\title{
pombalina
}

(8)

\section{Math as she is wrote}
Autor(es):
Cipra, Barry
Publicado por: Imprensa da Universidade de Coimbra; Gravida
URL persistente:
URI:http://hdl.handle.net/10316.2/32603
DOI:
DOI:http://dx.doi.org/10.14195/978-989-26-0485-5_1

Accessed : $\quad$ 26-Apr-2023 16:16:18

A navegação consulta e descarregamento dos títulos inseridos nas Bibliotecas Digitais UC Digitalis, UC Pombalina e UC Impactum, pressupõem a aceitação plena e sem reservas dos Termos e Condições de Uso destas Bibliotecas Digitais, disponíveis em https://digitalis.uc.pt/pt-pt/termos.

Conforme exposto nos referidos Termos e Condições de Uso, o descarregamento de títulos de acesso restrito requer uma licença válida de autorização devendo o utilizador aceder ao(s) documento(s) a partir de um endereço de IP da instituição detentora da supramencionada licença.

Ao utilizador é apenas permitido o descarregamento para uso pessoal, pelo que o emprego do(s) título(s) descarregado(s) para outro fim, designadamente comercial, carece de autorização do respetivo autor ou editor da obra.

Na medida em que todas as obras da UC Digitalis se encontram protegidas pelo Código do Direito de Autor e Direitos Conexos e demais legislação aplicável, toda a cópia, parcial ou total, deste documento, nos casos em que é legalmente admitida, deverá conter ou fazer-se acompanhar por este aviso. 
(Página deixada propositadamente em branco) 
MARIA PAULA SERRA DE OLIVEIRA

Coordenadora

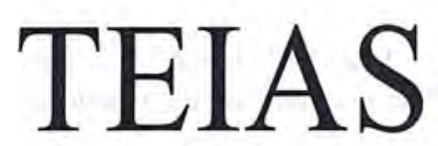

MATEMÁTICAS

Frentes na Ciência e na Sociedade 
(C) Gradiva - Publicações, L. da / Imprensa da Universidade de Coimbra, 2004 Coordenação editorial: Maria Paula Serra de Oliveira

Tradução: Artur Soares Alves

Carlota Isabel Leitão Pires Simões

Francisco José Craveiro de Carvalho

João Filipe Cortez Rodrigues Queiró

José Miguel Dordio Martinho de Almeida Urbano

Lia Sandra dos Santos

Mário da Silva Rosa

Paulo Eduardo Aragão Aleixo Neves de Oliveira

Revisão do texto: Isabel Pedrome

Capa: António Barros [Imprensa da Universidade. Coimbra], com imagem de

E. M. de Melo e Castro, "Fract 010 explod MC", Dezembro de 2003

[Fractal original gerado no Fractint com tratamento no Photoshop 7.0]

Infografia: Estúdios Estímulus [design]

Paginação: António Resende e Victor Hugo Fernandes

Impressão e acabamento: G.C. - Gráfica de Coimbra, L. da

Reservados os direitos para Portugal por:

Gradiva - Publicações, L. ${ }^{\text {da }}$ e Imprensa da Universidade de Coimbra

Gradiva - Publicações, L. ${ }^{d a}$

Rua Almeida e Sousa, 21, r/c, esq. • 1399-041 Lisboa

Telefs. $213974067 / 8 \cdot 213971357 \cdot 213953470$

Fax $213953471 \cdot$ Email: gradiva@ip.pt

URL: http://www.gradiva.pt

Imprensa da Universidade de Coimbra

Rua Antero de Quental, 195 • 3000-033 Coimbra

Telefs. 351239853110

Fax 3512398531 19 e-mail: fjrpress@ci.uc.pt

URL: http://www.imp.uc.pt

ISBN: 972-662-970-5

1." edição: Maio de 2004

Depósito legal n. ${ }^{\circ} 210431 / 04$

OBRA PUBLICADA COM O PATROCÍNIO DE:

CENTRO DE MATEMÁTICA DA UNIVERSIDADE DE COIMBRA DEPARTAMENTO DE MATEMÁTICA DA UNIVERSIDADE DE COIMBRA

FCT Fundação para a Ciência e a Tecnologia

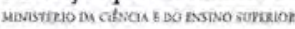




\section{Barry Cipra}

Colaborador da Society for Industrial

and Applied Mathematics

\section{Math as She is Wrote ${ }^{1}$}

Devo talvez começar por explicar o título deste texto. A ideia vem do título de um livro de frases português com tradução em inglês do século XIX, English as She is Spoke, de Pedro Carolino. O pobre Pedro não sabia inglês. Só tinha um manual com a versão francesa de expressões portuguesas e um dicionário de francês-inglês. A sua utilização em conjunto deu origem a alguns resultados excelentes, como o seguinte (primeiro em português, depois em inglês):

O sophista Zenon, um dos mais despejados humanos em sustentar paradoxos, negava, em certa occasião, na presença de Diogenes, a existencia do movimento. $O$ que ouvido pelo cynico, entra logo a cabriolar, e a correr diante dos ouvintes.

The sophist Zenon, the most hardy of all men at to sustain some paradoxes, was denied one day, before Diogenes, the existence of the motion. This was put him self immediately to make two or three turns in the session house.

A cavallo dado não se the olha para o dente.

A horse baared don't look him the tooth.

1 Tradução de João Filipe Queiró, professor do Departamento de Matemática, Universidade de Coimbra. 
Causa-me algum embaraço admitir que durante muito tempo o livro de Carolino foi a única obra literária portuguesa que conheci. Só há pouco tempo comecei a ler (em traduções) alguns dos muitos excelentes escritores que Portugal produziu. Estou especialmente enamorado de alguns poetas portugueses dos princípios do século $\mathrm{XX}$, incluindo Alberto Caeiro, Álvaro de Campos e Ricardo Reis ${ }^{2}$.

Normalmente, o livro de Carolino teria desaparecido, mas foi aproveitado como exemplo de humor involuntário pelo grande humorista da América, o escritor do século XIX Samuel Langhorne Clemens, mais conhecido pelo seu nome artístico, Mark Twain. «Ninguém pode acrescentar nada ao absurdo deste livro», escreveu ele numa introdução a English as She is Spoke. Graças a Twain, a vergonha de Carolino continuou à venda nas livrarias até aos dias de hoje.

$\mathrm{E}$ isto traz-nos a «Math as She is Wrote». A lição de Carolino é que corremos sempre um risco ao escrever com pretensões de autoridade sobre temas de que no fundo somos ignorantes. $\mathrm{O}$ que se diz pode facilmente parecer idiota a alguém que realmente sabe do assunto. Como jornalista da matemática, estou sempre consciente das oportunidades de dizer disparates. Lorde Northcliffe, o rei dos tablóides ingleses, apresentou o problema deste modo, ao definir jornalismo como «uma profissão que consiste em explicar aos outros o que ela própria não compreende».

Mais à frente darei alguns exemplos, de vários artigos recentes que escrevi sobre assuntos que pessoalmente não entendo. Mas vou começar com algumas observações gerais sobre a actividade de escrever.

De vez em quando perguntam-me qual o segredo da boa escrita. Quem me dera saber! Aprecio a posição de Fernando Pessoa sobre o assunto, no Livro do Desassossego: "Gosto de dizer. Direi melhor: gosto de palavrar. As palavras são para mim corpos tocáveis, sereias visiveis, sensualidades incorporadas.» Contudo, em matéria de conselhos úteis, o melhor que consigo dar-vos é que arranjem um revisor.

Não estou a brincar. Ter alguém que leia o que vocês escreveram $e$ corrija os problemas que o texto traga é uma das melhores maneiras de se tornarem bons escritores. Poucas pessoas o fazem, o que pode explicar

2 Para os leitores que não estejam familiarizados com a literatura portuguesa, há aqui uma brincadeira: Caeiro, Campos e Reis são todos o mesmo escritor, Fernando Pessoa, que escreveu também sob outros pseudónimos (chamava-lhes uheterónimos»), Falando aproximadamente, Pessoa é o Walt Whitman da poesia portuguesa. 
tanta prosa horrível que por aí anda, especialmente em revistas de matemática. Se querem um bom exemplo, tomem este mesmo artigo. O que estão a ler é essencialmente um primeiro rascunho (talvez a tradução em português ajude).

A figura 1, por exemplo, mostra o que Paul Zorn, o meu revisor de What's Happening in the Mathematical Sciences, fez a uma só página de um dos artigos que escrevi para um dos volumes. Está muito reduzida, espero até que ilegível - não quero que a leiam, apenas que percebam o espírito. Não há uma única frase que o Paul tenha deixado inalterada. $\mathrm{E}$, acreditem ou não, ainda somos bons amigos!

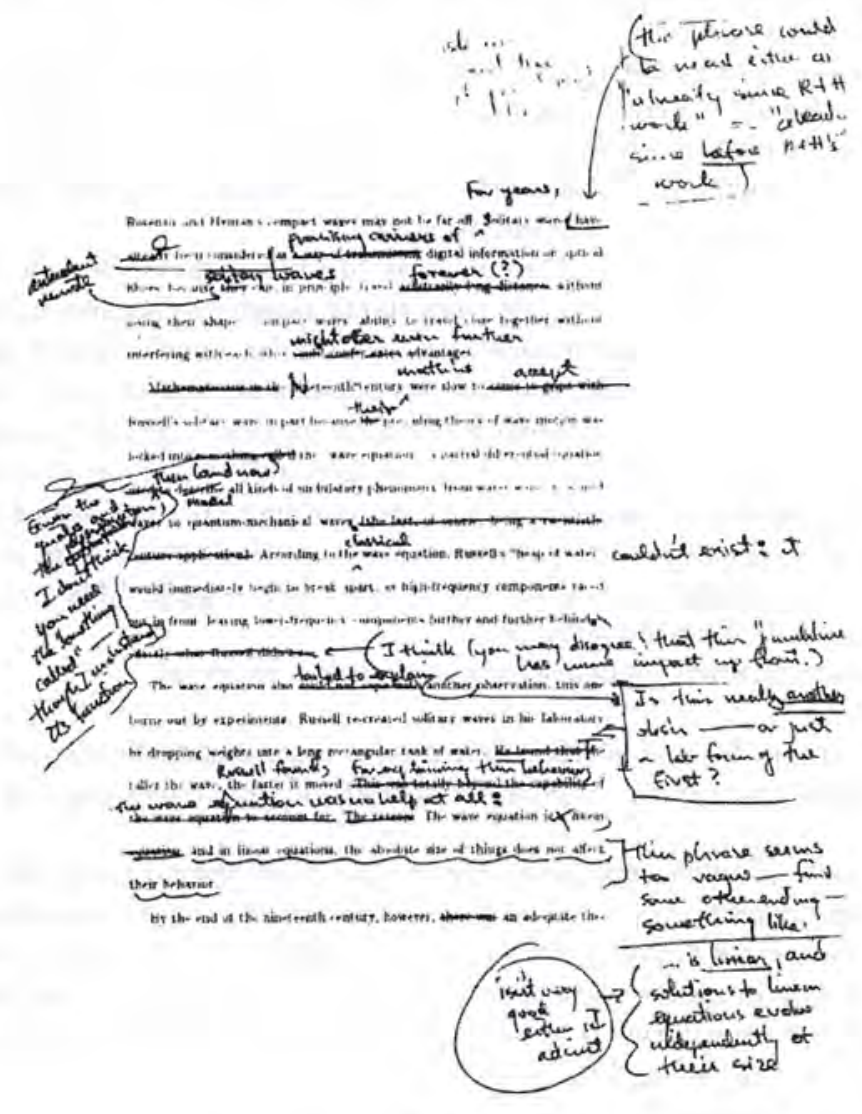

Fig. 1 
Outro bom conselho está contido num velho provérbio: «Uma figura vale mais que mil palavras». Na realidade, isso não é inteiramente verdade. Como freelancer, muitas vezes sou pago pelo espaço que preencho. Os editores não se importam que o preencha com palavras ou com figuras. De facto, até preferem figuras. Ora uma página numa revista como a Science leva cerca de mil palavras. E uma figura típica ocupa cerca de um quarto de página. Portanto, façam as contas: uma figura vale aproximadamente 250 palavras.

Mas voltemos à escrita sobre matemática e à maneira «certa» de fazer as coisas. Um dos truques é evitar o calão técnico - as palavras ou as frases que só têm significado para um pequeno círculo de pessoas. Falando por alto, há três típos de leitores: os matemáticos que já são especialistas no assunto sobre o qual se está a escrever, outros matemáticos que têm pelo menos uma compreensão geral do modo matemático de dizer as coisas e as massas matematicamente incultas. É para este último e vasto grupo que é preciso apontar se se quer popularizar a matemática. Mas usar esse truque pode ajudar, creio, a atingir também o segundo grupo, e mesmo, até certo ponto, o primeiro.

Vejamos um exemplo. Há uns anos (de facto em 1989), eu estava a escrever um artigo sobre um importante desenvolvimento na teoria das curvas elípticas relacionado com uma coisa chamada o grupo de Tate-Shafarevich. Esse desenvolvimento era uma técnica que permitiu aos especialistas em teoria dos números construir exemplos de curvas elípticas cujo grupo de Tate-Shafarevich é finito; a conjectura era (e continua a ser!) que o grupo de Tate-Shafarevich de qualquer curva eliptica é finito, mas, antes deste desenvolvimento, havia só um exemplo para o qual se sabia que a conjectura era verdadeira. Isto é muito interessante. Mas que diabo é um grupo de Tate-Shafarevich?

A minha primeira tentativa não teve muito sucesso:

O grupo de Tate-Shafarevich é uma estrutura algébrica que aparece no estudo de pontos racionais de uma curva elíptica.

Note-se que já tinha explicado antes o que é um «ponto racional». Esta explicação era má, e eu sabia-o. Assim, perguntei a um dos especialistas no assunto se me podia ajudar com uma explicação para leigos. Depois de pensar um momento, ele sugeriu o seguinte: o grupo de Tate-Shafarevich é o análogo para curvas elípticas do grupo das classes de ideais para corpos de números algébricos.

Ora bem, isto pode fazer todo o sentido para vocês. Na realidade, até fazia sentido para mim, porque a minha pós-graduação foi sobre teoria 
dos números e uma vez frequentei uma disciplina de teoria algébrica dos números. Contudo, penso que concordarão que não é provável que seja muito esclarecedor para as massas incultas - ou mesmo para grande parte das massas cultas.

Eis o que eu acabei por escrever:

O grupo de Tate-Shafarevich é o tapete para debaixo do qual os especialistas em teoria dos números varrem todas as dificuldades do cálculo de pontos racionais de curvas elipticas.

Não há dúvida de que isto não é uma definição, mas transmite a ideia que se pretende. Em particular, ajuda a explicar por que razão o tal desenvolvimento é tão importante: se o tapete é finito, só pode haver uma dificuldade finita no cálculo de pontos racionais.

Mesmo quando se escreve para especialistas, uma abordagem suave e não técnica pode ajudar. Em 1952 foi publicado um artigo intitulado «Dois teoremas sobre funções contínuas». Um dos teoremas dizia qualquer coisa como isto:

Sejam $f$ e $g$ funções contínuas de $[0,1]$ para $[0,1]$ tais que $f(0)=g(0)=0$ e $f(1)=g(1)=1$. Então existem funções contínuas $u$ e $v$ de $[0,1]$ para $[0,1]$ tais que $u(0)=v(0)=0, u(1)=v(1)=1$, e $f-u=g-v$.

É um bom teorema; no entanto, rapidamente foi esquecido. Quarenta anos depois foi publicado um artigo intitulado «O problema dos alpinistas». O teorema principal desse artigo foi apresentado de forma expositiva da seguinte maneira:

Dois alpinistas podem coordenar as suas subidas em lados opostos de uma montanha de forma a estarem sempre à mesma altitude.

Convenientemente analisado, este teorema é precisamente o mesmo que o outro.

Um último exemplo. Há uns anos escrevi um artigo sobre dinâmica dos fluidos, Nesse artigo precisava de explicar o que são as equações de Navier-Stokes. A minha primeira tentativa foi assim:

As equações de Navier-Stokes afirmam a conservação da massa e do momento para uma substância que se move com 
velocidade variável em cada ponto do espaço e é governada pelas leis de Newton sobre o movimento.

Isto veio do revisor com o seguinte comentário:

Pouco claro. O que é que entra nas equações e o que é que sai delas?

Acabámos por trocar alguma correspondência sobre o assunto. Muitas vezes o meu revisor manda a sua própria versão do que eu tentei explicar, e muitas vezes ela está completamente errada - ou é mesmo simplesmente disparatada. Isto pode ser frustrante (especialmente se já estamos em cima do prazo), mas de facto é extremamente útil. A tentação é rejeitar os comentários do revisor como delírios de um lunático ou equívocos de um cretino. Afinal a explicação original é perfeitamente clara, matematicamente clara. Mas o facto é que só é perfeitamente clara se o leitor for matemático. O revisor não é matemático e, mais pertinentemente, também o leitor pretendido não é matemático. E o revisor também não é um tolo (normalmente); o que ele é, na verdade, é o leitor mais atento que eu vou ter. Portanto, se ele não conseguir perceber o que eu escrevi, qual é a probabilidade de eu conseguir transmitir a ideia ao leitor médio?

Se a versão do revisor fosse directamente para a tipografia, as coisas estariam mal. $\mathrm{O}$ que faz o processo funcionar é que nós repetimos: eu tento perceber como é que ele conseguiu transformar a minha prosa claríssima numa coisa sem nexo, e depois tento reformular o texto de forma que faça sentido para um não-matemático (num certo caso, num artigo sobre qualquer coisa de teoria dos grafos, consegui finalmente perceber - porque ele me disse - que o revisor pensava que uma «aresta» de um grafo só podia ser uma das linhas entre vértices na periferia do grafo quando o desenhamos). No caso das equações de Navier-Stokes acabámos por chegar a uma formulação que ambos achámos ser razoavelmente clara:

As equações de Navier-Stokes apresentam as leis do movimento de Newton, que normalmente são aplicadas a objectos sólidos, em termos adequados para um fluido, em que a velocidade e a densidade podem variar de ponto para ponto no espaço.

Uma coisa a observar aqui é o aparecimento da palavra «fluido», que estava totalmente ausente do original! Claro que se pode objectar que a velocidade e a densidade também podem variar de ponto para ponto no 
espaço no caso de um sólido, mas é melhor ignorar estas questões mais subtis.

Para leitores com formação matemática, é evidente que para explicar as equações de Navier-Stokes só preciso de as escrever:

$$
\begin{gathered}
\frac{\partial u_{i}}{\partial t}+\sum_{j=1}^{n} u_{j} \frac{\partial u_{i}}{\partial x_{j}}=v \Delta u_{i}-\frac{\partial p}{\partial x_{i}} \\
\nabla \cdot u=0 \\
\mathrm{v}=\text { viscosidade }
\end{gathered}
$$

Está claro?

A propósito destas equações faço referência à seguinte conjectura: se $u(x, 0)$ for «suave», então $u(x, t)$ é suave para todos os $t \geq 0$.

Se conseguirem provar a verdade (ou a falsidade) desta conjectura, têm uma possibilidade de chegar à fama e à fortuna. Ela é um dos sete problemas matemáticos fundamentais incluídos em 2000 pelo Clay Mathematics Institute num conjunto cuja solução seria premiada com um milhão de dólares. Os outros referem-se à equação de Yang-Mills (em teoria quântica dos campos), à hipótese de Riemann, à conjectura de Poincaré, à conjectura de Hodge, à conjectura de Birch-Swinnerton-Dyer e ao problema $\mathrm{P} v s$. NP em computação teórica. Podem ver-se pormenores na página do instituto em www.claymath.org.

Vou tentar descrever o último, o problema P vs. NP, porque é um bom exemplo de um problema matemático difícil que surgiu por causa dos computadores.

P denota a classe de problemas que podem ser resolvidos em «tempo polinomial». Isto é uma forma técnica de dizer problemas «fáceis». Um exemplo é o problema da busca. Por exemplo, 101 aparece na lista seguinte?

$$
\begin{aligned}
& 1011,10,1,1101,11,1010,111,110,1,10101,1010,110, \\
& 1001,1011,10100,110101,1011,10001,11,10110,110, \\
& 10,1011
\end{aligned}
$$

Este problema é de tempo polinomial porque o tempo que demora resolvê-lo é limitado por uma potência do tamanho do problema, que neste caso é o número de algarismos na lista. 
NP, por seu turno, representa a classe de problemas que podem ser resolvidos em «tempo polinomial não determinista». Isto é uma forma técnica de dizer que são problemas «dificeis» (embora a própria palavra dificil seja usada num sentido técnico). Mais precisamente, um problema NP é uma pergunta de resposta $\operatorname{sim}$ /não em que a resposta «sim» pode ser verificada por um cálculo em «tempo polinomial». Um problema NP famoso é a factorização. Por exemplo, pode mostrar-se facilmente que a pergunta «Será que o número $5493216597273416 \ldots 117$ tem um factor primo menor que $427972417 \ldots 271$ ?» tem a resposta «sim», se conhecermos - ou adivinharmos - o factor primo apropriado. É aqui que entra o não-determinismo.

Esta definição é curiosamente assimétrica, porque uma resposta «não» a um problema NP pode ou não ser fácil de verificar. A classe dos problemas em que a resposta «não» é fácil de verificar mas «sim» pode ou não sê-lo chama-se co-NP. A questão da primalidade (por exemplo, o número $5493216597 \ldots 117$ é primo?) pertence a essa classe. Na teoria da complexidade computacional há uma hierarquia de classes de complexidade que incluem P, NP e co-NP. Uma pequena parte da hierarquia está na figura 2 .

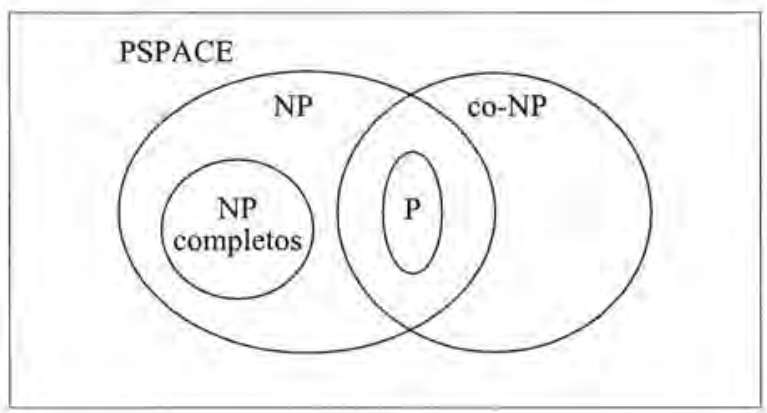

Fig. 2

A classe PSPACE consiste nos problemas computacionais que podem ser resolvidos usando uma quantidade polinomial de memória. A razão por que P está na intersecção de P com co-NP é óbvia: é sempre possível responder a uma pergunta em $\mathrm{P}$ (e nem é preciso adivinhar). De facto, foi conjecturado que P é toda a intersecção de NP com co-NP. Contudo, a conjectura famosa, uma das sete do Instituto Clay, é que NP é maior do que $\mathrm{P}$ - isto é, que existem problemas que são mesmo difíceis. 
O balão na figura com a Iegenda «NP completos» precisa de mais algumas explicações. De modo simplificado, trata-se de problemas que podem ser usados como "chave» para todos os outros na classe NP. Um exemplo é o clássico problema do caixeiro-viajante. O que isto significa é que qualquer problema na classe NP - a factorização, por exemplo - pode, com uma quantidade polinomial de trabalho, ser reformulado como um problema do caixeiro-viajante. A consequência disto é que, se se vier a concluir que existe um algoritmo de tempo polinomial para o problema do caixeiro-viajante, tudo o que está em NP cai dentro de P. Dito de outra forma, se se acredita (ou se supõe) que é verdadeira a conjectura de que $\mathrm{P}$ não é igual a NP, então pertencer à classe dos problemas NP completos é «prova» de que um problema é verdadeiramente difícil.

Há muitas centenas de exemplos de problemas NP completos, alguns dos quais vou referir daqui a pouco. No entanto, começo por mencionar dois problemas que estão em NP mas que não se sabe se são NP completos: a factorização e o problema do isomorfismo de grafos. Na verdade, conjectura-se que estes não são NP completos, apesar de se acreditar que ambos estão fora de $\mathrm{P}$.

A lista de problemas NP completos tem vindo a crescer com regularidade desde que o conceito foi definido e os primeiros exemplos dados, no princípio da década de 70. Em 2000, Sorin Istrail, dos Sandia National Laboratories (actualmente na Celera Genomics), acrescentou um exemplo impressionante: o modelo de Ising tridimensional de um vidro de spin. Trata-se de um problema em mecânica estatística que surgiu nos anos 20, quando Ernst Ising estudou o modelo «ferromagnético» de dimensão 1. Em geral, o modelo de Ising considera uma rede de átomos $i$, cada um dos quais pode estar em um de dois estados, $\sigma_{i}= \pm 1$. Entre átomos adjacentes $i$ e $j$ há um «coeficiente de emparelhamento» $J_{i j}$ para a interacção dos dois átomos. Este coeficiente entra no "hamiltoniano» do sistema,

$$
H(\sigma)=-\sum J_{i j} \sigma i \sigma j / k \mathrm{~T}
$$

onde a soma é feita para todos os pares de átomos adjacentes, $k$ é a constante de Boltzmann e $T$ é a temperatura. E temos a importante «função de partição»,

$$
Z(T)=\sum_{\sigma} e^{-H(\sigma)}=\sum_{E} n(E) e^{-E},
$$

onde a primeira soma é feita para todas as configurações possíveis do sistema (isto é, atribuições de \pm 1 aos átomos individuais) e a segunda soma 
é feita para os correspondentes valores do hamiltoniano. Um problema computacional (de facto há vários) é encontrar um «estado básico», uma configuração de energia mínima.

Para o modelo ferromagnético original de Ising isso não é problema: ser ferromagnético significa que todos os $J_{i j}$ são iguais a 1 e o estado básico obtém-se fazendo todos os $\sigma_{i}$ tomar o mesmo valor (não importa se 1 se -1). O caso antiferromagnético é igualmente fácil, mas o caso de um vidro de spin, em que pode ocorrer uma mistura arbitrária de valores para os coeficientes de emparelhamento, é outra história. Mesmo assim, é trivial para uma rede de dimensão 1 , e «fácil» para redes bidimensionais, no sentido de que pertence à classe $\mathrm{P}$ (a demonstração para o caso bidimensional, no entanto, não é nada fácil!). Mas mais nada. Istrail mostrou que o problema do vidro de spin é NP completo para redes não planares. Isto inclui o problema em dimensão 3, mas também casos bidimensionais em que, além das interacções com o vizinho mais próximo, são incluidas interacções com os segundos mais próximos.

O modelo de Ising é importante no estudo de transições de fase, que são mudanças bruscas de estado ocorrendo a certa temperatura crítica, como o congelamento da água a 0 graus Celsius. Estranhamente, os especialistas em computação descobriram há pouco tempo alguns fenómenos análogos na sua própria área: certos problemas NP parecem mudar de extremamente fáceis para extremamente difíceis quando o problema atinge um certo tamanho crítico.

O exemplo principal, estudado do ponto de vista das transições de fase pela primeira vez por Scott Kirkpatrick (um físico) e Bart Selman (um especialista em computação), é um problema NP completo conhecido por 3-SAT. Curiosamente, o 3-SAT é o problema NP completo original, o primeiro exemplo alguma vez encontrado. O problema diz respeito à "satisfactibilidade» de expressões lógicas como

$$
\left(x_{1} \vee x_{2} \vee \overline{x_{3}}\right)\left(\overline{x_{1}} \vee x_{3} \vee x_{4}\right) \wedge \ldots \wedge\left(x_{15} \vee \overline{x_{23}} \vee x_{102}\right),
$$

onde há, digamos, $M$ condições, cada uma com três variáveis boolianas, e um total de, digamos, $N$ variáveis boolianas $\left\{x_{1}, x_{2}, \ldots, x_{N}\right\}$ (os símbolos ^ e $\vee$ significam «ou» e «e», e $\bar{x}$ significa «não $x$ »). Diz-se que a expressão é satisfazível se alguma atribuição de valores lógicos, Verdadeiro e Falso, às variáveis torna a expressão verdadeira. Mais geralmente, o problema $k$-SAT diz respeito a expressões em que aparecem $k$ variáveis em cada condição.

Kirkpatrick e Selman estudaram transições de fase no problema $k$-SAT para $k=3,4,5$ e 6 (o caso $k=2$ tem menos interesse porque o problema 
2-SAT pertence à classe $\mathrm{P}$ ). $\mathrm{O}$ que eles descobriram foi que, quando $\mathrm{o}$ quociente $M / N$ aumenta, a probabilidade de que uma expressão $k$-SAT gerada aleatoriamente não seja satisfazível passa de perto de $0 \%$ para quase $100 \%$, com uma transição assimptoticamente brusca num valor que depende de $k$. Em particular, para $k=3$, o valor crítico é $M / N \approx 4.17$, como indicado na figura 3.

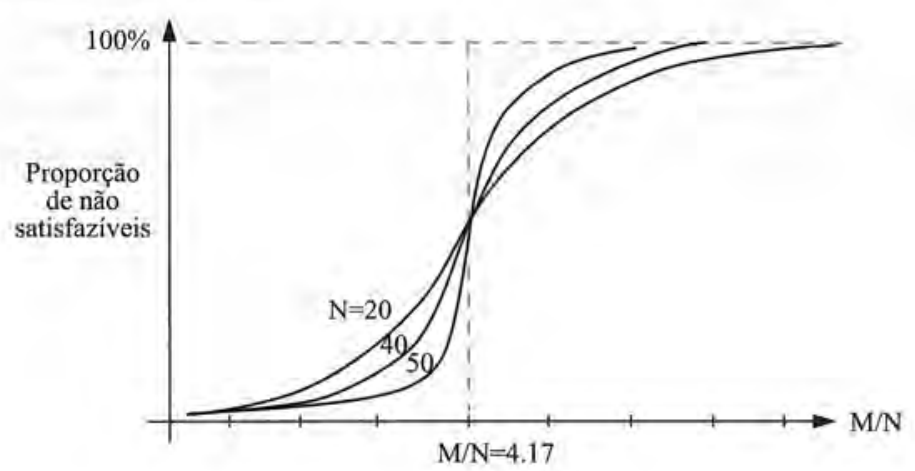

Fig. 3

Além disso, a quantidade de trabalho computacional exigida para dizer se uma expressão é ou não satisfazível parece ter um pico nas vizinhanças do mesmo valor crítico (figura 4 ).

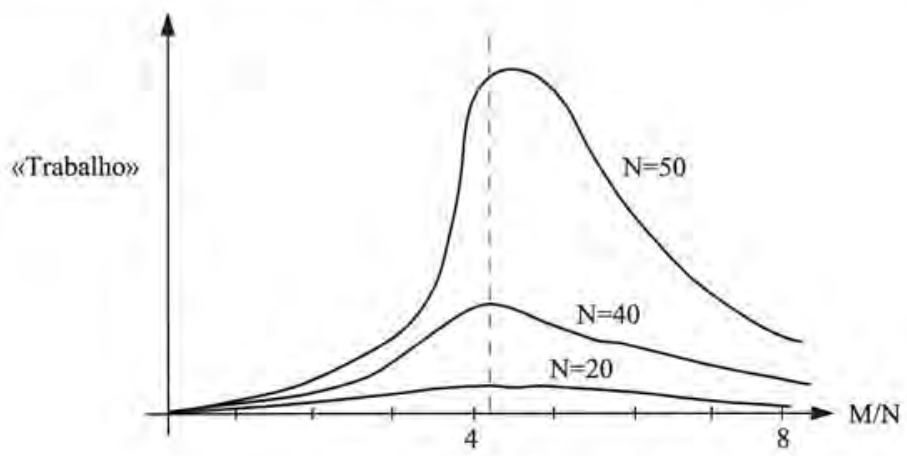

Fig. 4

Resta perceber o que significa exactamente isto, mas muitas pessoas estão actualmente a investigar a «mecânica estatística» de problemas computacionais. 
Bom, penso que chega. Disse-vos o que sei sobre a escrita (não muito) e dei-vos alguns exemplos do que acontece quando tentamos explicar assuntos que pessoalmente não entendemos. Espero, todavia, ter-vos proporcionado alguma compreensão de certas coisas e estimulado o vosso interesse nelas. Se quiserem aprender mais, uma boa maneira de o fazer é irem à Internet e introduzirem expressões como «istrail ising npcomplete» ou «kirkpatrick selman k-sat» no vosso motor de busca favorito (o meu é o google.com) e ver onde elas vos levam. Podem também tentar uma busca sobre «barry cipra». Mas não vale a pena verem www.cipra.com ou www.cipra.org - o que vão encontrar é o California Institute for Public Risk Assessment, num caso, e a Commission International pour la Protection des Alpes no outro! 
(Página deixada propositadamente em branco) 


\section{$\begin{array}{llllll}\text { C I E } & \mathbf{N} & \mathbf{C} & \mathbf{A}\end{array}$}

mitiram realizar com sucesso tarefas tão distintas como a programação de um voo a Marte, a previsão de resultados eleitorais, a explicação do funcionamento de alguns mecanismos do sistema nervoso, ou a abordagem critica de obras de arte e de textos literários. Da ciência à sociedade, dos grandes avanços técnicos à solidez de uma argumentação lógica, a Matemática constrói teias de uma imensa flexibilidade resultante do carácter universal da sua linguagem.

Neste livro, personalidades de diferentes universos dão o seu testemunho sobre a forma como usam as teias matemáticas para tecer a sua própria visão do mundo.

Maria Paula Serra de Oliveira é professora de Matemática na Faculdade de Ciências e Tecnologia da Universidade de Coimbra. 